\title{
Characteristics of Dark Current and Photocurrent in Superlattice Infrared Photodetectors
}

\author{
Wen-Hsing Hsieh ${ }^{\mathrm{a}}$, Chun-Chi Chen ${ }^{\mathrm{a}}$, Jet-Ming Chen ${ }^{\mathrm{a}}$, Yuen-Wuu Suen ${ }^{\mathrm{b}}$ and Chieh-Hsiung Kuan ${ }^{* a}$ \\ ${ }^{a}$ Department of Electrical Engineering, National Taiwan University, Taipei, Taiwan, 10617 \\ ${ }^{\mathrm{b}}$ Department of Physics, National Chung-Hsing University, Taichung, Taiwan, 402
}

\begin{abstract}
The characteristics of a superlattice infrared photodetector, which has a 20-period GaAs/AlGaAs superlattice embedded between two AlGaAs current blocking layers are investigated. We propose a model to explain the conduction of dark current and photocurrent. In particular, the interesting feature of this detector, which can also be explained by our model, is bias redistribution due to the background photocurrent. With the background radiation incident upon the detector, the blocking layers are tilted up for $126 \mathrm{meV}$ at zero bias. The electrons are all confined by the tilt-up blocking layers and cannot tunnel through them. As the external bias increases, $70 \%$ of the voltage is added on the rear blocking layer and attempts to decrease its barrier height. Especially at $-0.18 \mathrm{~V}$, the barrier is flat and the barrier height is $16.5 \mathrm{meV}$ higher than the bottom of the second miniband. In this case, only those photoelectrons with high enough energy can pass the blocking layer. As the external bias increases up to $-0.33 \mathrm{~V}$, the rear blocking layer is tilted down for $105 \mathrm{meV}$. The blocking layer seems to be transparent to all photoelectrons to tunnel through. In addition, the photo responsivity due to the short wavelength excitation indicates that the photoelectrons do have the inelastic relaxation of the energy along the growth direction.
\end{abstract}

Keywords: Superlattice infrared photodetector, current blocking layer, dark current, and photocurrent.

\section{INTRODUCTION}

Long wavelength infrared detection using intersubband transitions in quantum well structures has been under intensive investigation. ${ }^{1 .-5}$. Such multiple quantum well detectors are characterized by a thick active region that contains usually 30 to 50 quantum wells. ${ }^{6}$. In contrast to the multiple quantum well detectors, the single quantum well detector has the advantages of saving the material and reducing the operating bias. However, the bias distribution on the barrier region is not stable because of the space charge accumulation. Therefore, we propose a novel detector which is similar to the single quantum well structure but with the superlattice rather than the quantum well to absorb infrared radiation. The device is expected to be stable due to the large capture rate in the superlattice than that in the quantum well, and the operation bias is much smaller than that of the conventional multiple quantum well infrared photodetector (QWIP) due to the narrow barrier thickness of the superlattice structure.

The characteristics of the dark current and photocurrent in our detector will be shown in this paper and a simple model is proposed to describe them. One component of the dark current is the thermionic emission (TE) current and the other is the thermally assisted tunneling (TAT) current. ${ }^{7.9}$. The simulation for the dark current agrees well with the experiment data in this paper. With infrared radiation, the applied voltage is almost dropped on the rear blocking layer in order to supply the dark current plus the background photocurrent. In addition, the photo responsivity due to the short wavelength excitation indicates that the photoelectrons do have the inelastic relaxation of the energy along the growth direction.

This paper is organized as follow. Section 1 is a brief introduction and section 2 shows the sample structure and the device process. The characteristics of the dark current and photocurrent will be shown in section 3 and section 4 . In section 3 , we will present a simple model to describe the physical mechanisms of the dark current. The fitting result matches well with the experiment. Similarly we also derive a formula to describe the photocurrent and demonstrate that the formula can describe the experimental results of responsivity versus bias for a monochromatic radiation and spectral response in section 4 . The final concusion is given in section 5 .

*Correspondence: Email: kuan@cc.ee.ntu.edu.tw; Telephone: +886-2-23635251 ext. 439; Fax: +886-2-23671909.

Physics and Simulation of Optoelectronic Devices IX, Yasuhiko Arakawa, Peter Blood, Marek Osinski, Editors, Proceedings of SPIE Vol. 4283 (2001) @ 2001 SPIE · 0277-786X/01/ $\$ 15.00$ 


\section{SAMPLE STRUCTURE}

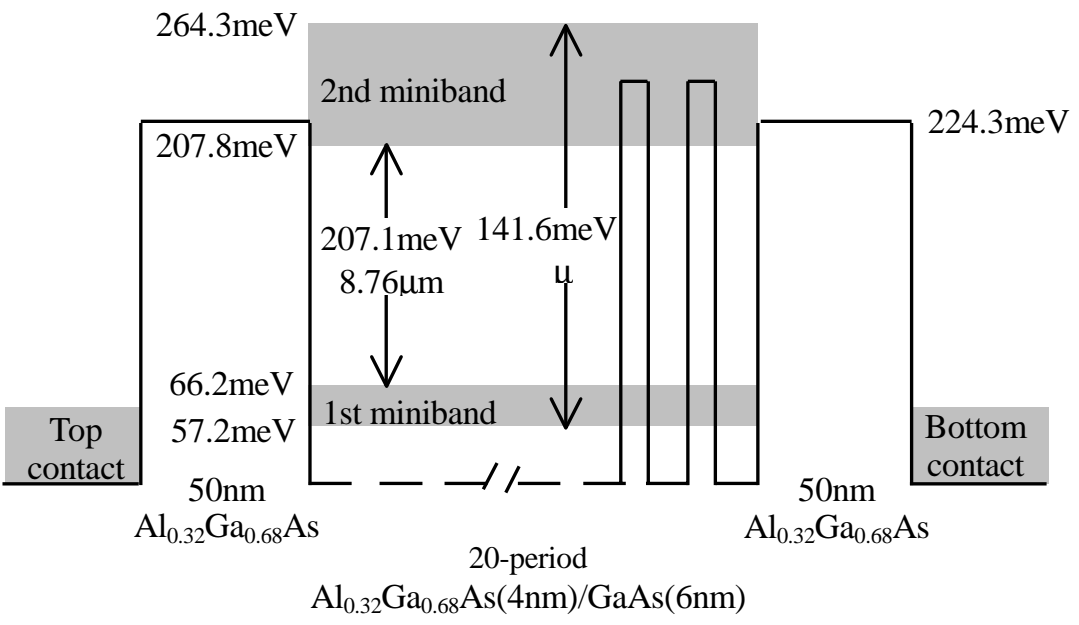

Fig. 1 Schematic energy band structure diagram without extend bias. The GaAs well region of the superlattice is doping with $1 \times 10^{17} \mathrm{~cm}^{-3}$ of $\mathrm{Si}$.

The schematic band diagram of our detector is shown in Fig. 1 where the superlattice structure is designed to absorb infrared radiation by the miniband transitions of electrons. The miniband energies are calculated with the effective mass approximation and the Kronig-Penny model. ${ }^{10}$. The first and second miniband of a 20 -period $\mathrm{GaAs}(6 \mathrm{~nm}) / \mathrm{Al}_{0.32} \mathrm{Ga}_{0.68} \mathrm{As}(4 \mathrm{~nm})$ superlattice ranges from 57.2 to $66.2 \mathrm{meV}$ and 207.8 to $264.3 \mathrm{meV}$ correspondingly. ${ }^{11 .}$ Therefore, the designed peak absorption wavelengths are at $5.99 \mu \mathrm{m}$ and $8.76 \mu \mathrm{m}$ corresponding to transitions at zone center and edge respectively. In addition, the barrier height of the front and rear blocking layers is both $16.5 \mathrm{meV}$ above the bottom of the second miniband. The superlattice is doped with $1 \times 10^{17} \mathrm{~cm}^{-3}$ of Si to provide electrons for miniband transition and the ohmic contact layers are doped with $1 \times 10^{18} \mathrm{~cm}^{-3}$ of Si.

The current blocking layer is introduced to reduce the dark current originated from the conduction of the electrons in the first miniband of the superlattice. The energy of the blocking layer is designed to be high enough so that the dark current is suppressed more efficiently than the photocurrent that is mainly caused by the photoelectrons of in the second miniband tunneling through the blocking layer.

The samples were processed into $145 \times 145(\mu \mathrm{m})^{2}$ mesa by standard lithography and wet chemical etching. AuGeNi/Au was evaporated on top and bottom to form the ohmic contact, and the detector was polished into $45^{\circ}$ on the substrate facet for light coupling.

The I-V curves for the dark current and photocurrent in our detector are measured and analyzed, and the bias dependence of the responsivity at various wavelength are also measured in order to understand the transport behavior of the second miniband.

\section{MODEL AND EXPERIMENT-PART I}

\subsection{Dark Current Model}

We first present a simple model to describe the physical mechanisms of the dark current in our detector. Figure 2 demonstrates the electron path across the detector for the dark current. The injected electrons from the top contact pass the front blocking layer and enter into the second miniband of the superlattice. The superlattice structure is assumed to be long enough so that the electrons are relaxed into the first miniband. Similar to those electrons from the top contact, the injected electrons from the superlattice pass the rear blocking layer and enter into the bottom contact. The currents caused by the electrons through the front and rear blocking layers must be equal. Although the densities of states in the top contact and the superlattice are not the same, the voltage drops on the front and rear blocking layers are assumed to be the same for the dark current. Due to the doping density, no voltage drop in the superlattice is also expected. 


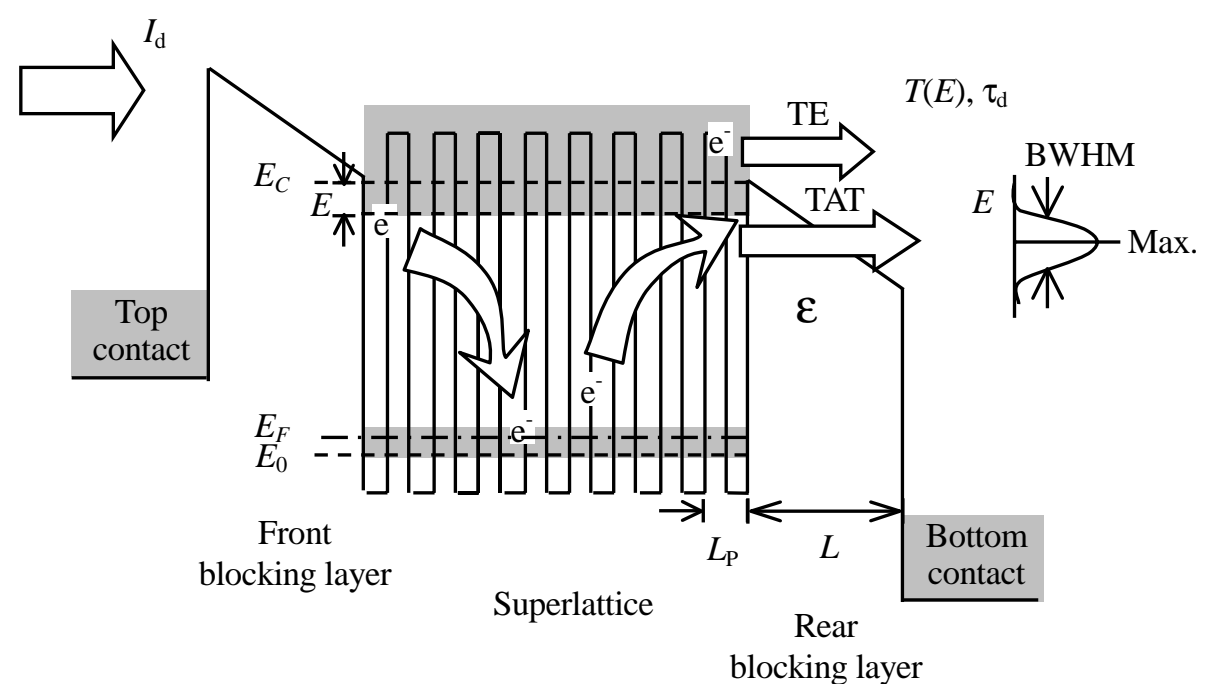

Fig. 2 The electron path across the detector for the dark current.

Our emphasis is made on the dark current generated by those injected electrons from the superlattice passing the rear blocking layer. In the superlattice, the electron state is quartzite in the growth direction and move freely in the plane perpendicular to the growth direction. Electrons with momentum perpendicular to the growth direction may be scattered by impurities or phonons and pass the blocking layer with the transmission Probability $T(E)$ where E is their individual total energy instead of the ground-state energy. In this case, we can use a simple formula to express the dark current $I_{\mathrm{D}}$,

$$
I_{D}=\int \frac{e m^{*}}{\pi \hbar^{2} L_{\mathrm{p}}} f(E) \cdot A L_{\mathrm{p}} \cdot T(E) \cdot \frac{1}{\tau_{\mathrm{d}}} d E
$$

where $m^{*}$ is effective mass of GaAs, $L_{\mathrm{p}}$ is the length of each period in superlattice, $f(E)$ is the Fermi-Dirac distribution, $\tau_{\mathrm{d}}$ is the scattering time by the impurities or phonons to transfer their energy along the superlattice plane into the growth direction, and $A$ is the cross-section area of the detector.

Theoretically, the dark current consists of the thermionic emission current $I_{\mathrm{TE}}$ and the thermally assisted tunneling current $I_{\mathrm{TAT}} \cdot{ }^{12-14 .}$ The former is contributed by those electrons with $E>E_{\mathrm{c}}$, so that the transmission probability $T(E)$ is equal to one. The latter is attributed to the other electrons with $E<E_{\mathrm{c}}$ and with the assistance of the electric field to tunnel through the blocking layer. Using the W.K.B. method, we may write the two current components as

$$
\begin{aligned}
I_{\mathrm{TE}} & =\int_{E_{\mathrm{c}}}^{\infty} \frac{e m^{*} A}{\pi \hbar^{2} \tau_{\mathrm{d}}} \cdot f(E) \cdot d E \\
& =\frac{e m^{*} A}{\pi \hbar^{2} \tau_{\mathrm{d}}} \cdot k_{B} T \exp \left(-\frac{E_{\mathrm{A}}}{K_{\mathrm{B}} T}\right),
\end{aligned}
$$

and

$$
\begin{aligned}
I_{\mathrm{TAT}} & =\int_{E_{0}}^{E_{c}} \frac{e m^{*} A}{\pi \hbar^{2} \tau_{\mathrm{d}}} \cdot f(E) \cdot T(E) \cdot d E \\
& =\frac{e m^{*} A}{\pi \hbar^{2} \tau_{\mathrm{d}}} \cdot \sqrt{\ln 2 \cdot k_{B} T \frac{\hbar^{2}}{2 m^{*}}\left(\frac{e \varepsilon}{k_{B} T}\right)^{2}} \cdot \exp \left(-\frac{E_{\mathrm{A}}}{K_{\mathrm{B}} T}+\frac{\Delta E_{\mathrm{B}}}{k_{\mathrm{B}} T}\right),
\end{aligned}
$$




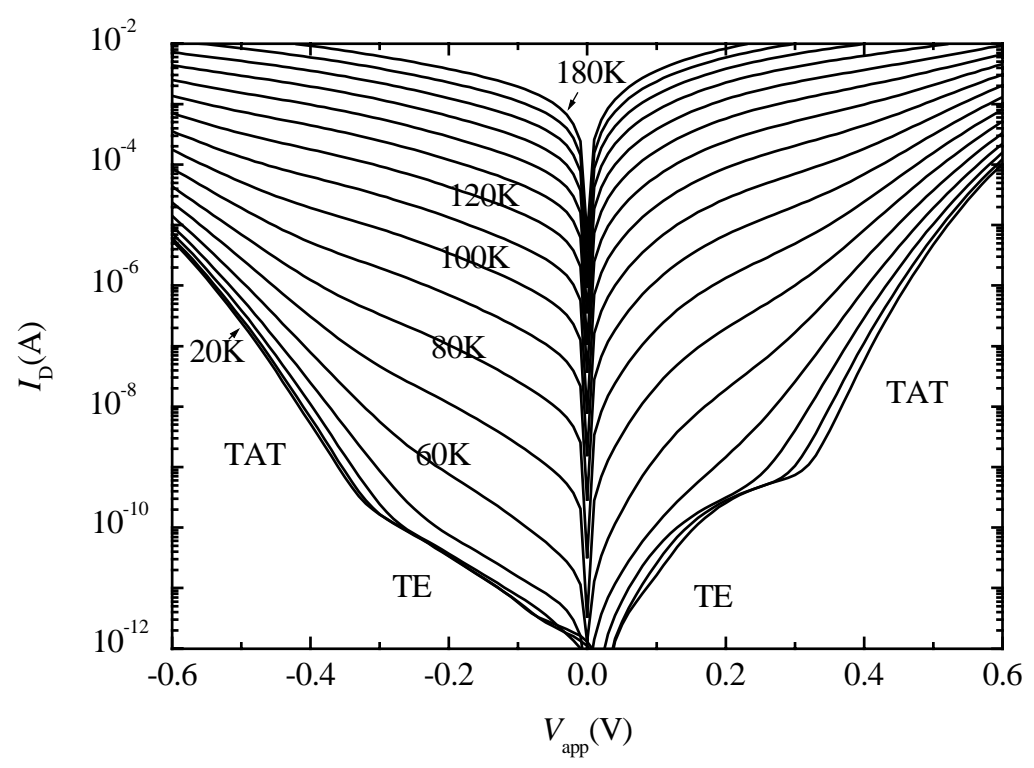

Fig. 4 The dark-current $I-V$ characteristics at various temperatures.

where $E_{\mathrm{A}}=E_{\mathrm{c}}-E_{\mathrm{F}}-\beta V_{\text {app }}$ is the activation energy, $\varepsilon=V_{\text {app }} / L$ is the electric field in the current blocking layer with $V_{\text {app }}$ and $L$ are the external bias and the thickness of the current blocking layer, $\Delta E_{\mathrm{B}}$ is a main factor to describe the TAT process. The factor $\beta V_{\text {app }}$ is to explain the rise of the Fermi level due to the accumulation of electrons at the interface between the superlattice and the blocking layer under the external applied bias. In the derivation, we have assumed that $\tau_{\mathrm{d}}$ is a constant and independent of $E$ for simplicity.

As shown in Fig. 2, the energy distribution of those electrons passing the blocking layer with thermally assisted tunneling can be described with the peak magnitude and the energy bandwidth of the half magnitude. The total number of the electrons is approximately equal to the product of the peak magnitude and the bandwidth. The peak magnitude is equivalent to find the maximum value of $f(E) \cdot T(E)$ in Eq.(3) where $T(E)$ is given by W.K.B. method as

$$
T(E)=\exp \left(-\sqrt{\frac{2 m_{\mathrm{B}}^{*}}{\hbar^{2}}} \cdot \frac{4}{3 e \varepsilon}\left(E_{\mathrm{c}}-E\right)^{\frac{3}{2}}\right) .
$$

In the formula, $m_{\mathrm{B}}$ is the barrier effective mass. The Fermi-Dirac distribution $f(E)$ is approximately $\exp \left(-\left(E-E_{\mathrm{F}}\right) / k_{\mathrm{B}} T\right)$, i.e., $E-E_{\mathrm{F}}$ is assumed to be much lager than $k_{\mathrm{B}} T$. The energy bandwidth of the half magnitude can be found with $f(E) \cdot T(E)$ equal to the half of the peak magnitude. The final result gives $\Delta E_{\mathrm{B}}$ as

$$
\Delta E_{B}=\frac{\hbar^{2}}{24 m_{\mathrm{B}}^{*}}\left(\frac{e \mathcal{E}}{k_{\mathrm{B}} T}\right)^{2} .
$$

\subsection{Experiment and Analysis for Dark Current}

Experimentally, the current-voltage (I-V) characteristics were measured by using a Keithley 236 source measurement unit. The sample was mounted on the cold head of an APD DMX-20 closed-cycle cryostat to control the sample temperature. A Si-diode temperature sensor was also mounted near the sample on the same cold head. We have recorded and controlled the sample temperature by using the Si-diode sensor. 


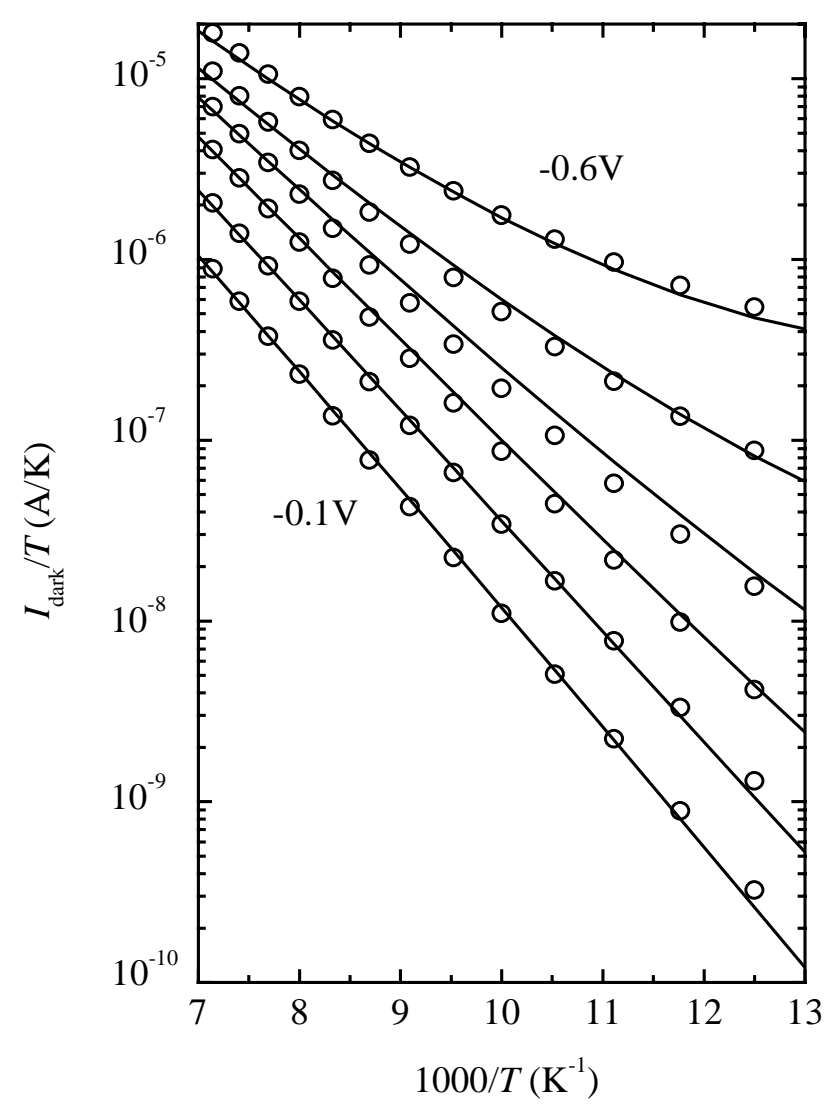

Fig. 5 Plot the dark current (scatter curve) as a function of inverse $k_{\mathrm{B}} T$ at -0.1 to $-0.6 \mathrm{~V}$ bias voltages (solid curve).

The dark-current $I-V$ characteristics at various temperatures are shown in Fig. 4 . For the detector at 20k, the dark current can be obviously separated into the two regimes. In the low bias regime, the dark current seems to increase less sensitively to the applied bias. This is attributed to the thermionic emission (TE) process of the electrons through the blocking layer. In the second regime, the dark current is more sensitive to the applied bias. Actually this is the primary characteristic of the thermally assisted tunneling (TAT) mechanism.

To use our model to explain the dark current $I_{\mathrm{D}}$, especially the trend of the activation energy, we plot $I_{\mathrm{D}} / T$ as a function of inverse $T$ at negative-bias voltages in a semi-log scale. The result is shown as the open circle in Fig. 5. In terms of Eqs. (2) (5), the function can be written as

$$
\ln \left(\frac{I_{d}}{T}\right)=-\frac{E_{\mathrm{A}}}{k_{\mathrm{B}} T}+\ln \left[1+\frac{\alpha V}{T^{3 / 2}} \cdot \exp \left(\frac{\Delta E_{\mathrm{B}}}{3 k_{\mathrm{B}} T}\right)\right]+\ln \frac{e m^{*} A}{\pi \hbar^{2} \tau_{\mathrm{d}}},
$$

where $\alpha=\sqrt{\ln 2 \cdot k_{B} \frac{\hbar^{2}}{2 m^{*}}\left(\frac{e}{k_{B}}\right)^{2}}$.

The solid curves in Fig. 5 are the fitting results. The main fitting parameters are $\alpha$ and $\beta$, which are equal to $8616 \mathrm{~K}^{3 / 2} \mathrm{~V}^{-1}$ and $46.67 \mathrm{meV} / V_{\text {app }}$ derived from experimental data. Theoretically $\alpha$ is equal to $8111 \mathrm{~K}^{3 / 2} \mathrm{~V}^{-1}$ and agrees with the experimental results. The activation energy $E_{\mathrm{A}}$ is equal to $154 \mathrm{meV}$ at $V=0$ and is consistent with the design value $150 \mathrm{meV}$. The accumulated space charge at the interface between superlattice and the rear blocking layer may cause the Fermi level to rise about $28 \mathrm{meV}$ at $V_{\text {app }}=-0.6 \mathrm{~V}$. This is equivalent to extra charge density $7.8 \times 10^{17} \mathrm{~cm}^{-3}$ there. 


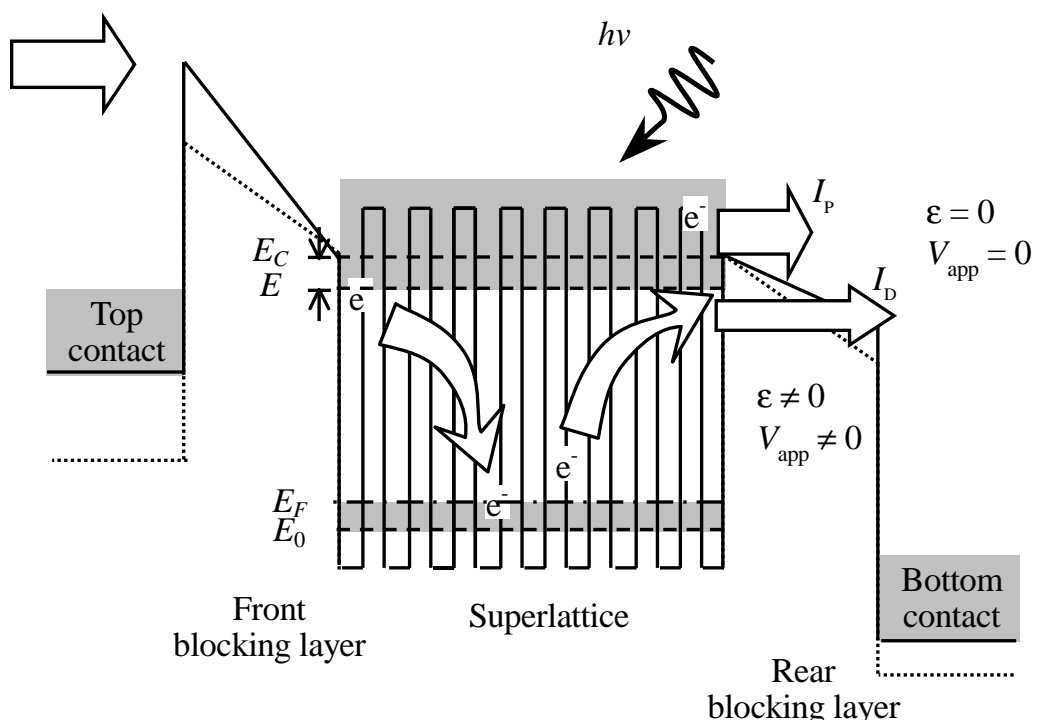

Fig. 6 The electron path and the bias distribution with dark current (dash line) and photocurrent (solid line).

The sensitivity of $I_{\mathrm{TE}}$ to the external bias is due to the factor of $\beta V_{\text {app }}$ while that of $I_{\mathrm{TAT}}$ is due to $\Delta E_{\mathrm{B}}$. Since $\Delta E_{\mathrm{B}}$ is proportional to $V_{\text {app }}^{2}, I_{\mathrm{TAT}}$ is more sensitive to the external bias than $I_{\mathrm{TE}}$.

\section{MODEL AND EXPERIMENT-PART II}

\subsection{Photocurrent Model}

In this section, we first derive a formula to describe the photocurrent and then demonstrate the experiment results of responsivity versus bias and spectral response. In the end, we discuss the agreement between the experimental results and our formula.

With infrared radiation incident upon the sample, the electrons are excited from the first miniband into the second miniband. There may exist some relaxation mechanism, to cause the energy of the photoelectron along the growth direction to decrease.

For example, the impurities or phonons in the superlattice may scatter the photoelectrons and transfer their energy. Therefore, the generation rate $G(E)$ of the photoelectrons by the infrared radiation may be a function of energy $E$ where $E$ is between the second miniband of the superlattice. For simplicity, we assume the photoelectron with energy $E$ has a lifetime $\tau_{f}$ and velocity $v$ to oscillate between the two blocking layers where $\tau_{\mathrm{f}}$ and $v$ are both independent of the energy. Under these circumstances, the photocurrent $I_{\mathrm{P}}$ can be written as

$$
I_{P}=\int e G(E) \tau_{\mathrm{f}} v \cdot T(E, \varepsilon) d E
$$

where $T(E, \mathcal{E})$ is the transmission probability of the photoelectrons through the blocking layer. Again using the W.K.B. method gives $T(E, \mathcal{E})$ as Eq. (4).

With only dark current existing, 50\% of the applied voltage drops on the front blocking layer and 50\% does on the rear blocking layer as shown in Fig. 2. With additional background photocurrent, most of the applied voltage is dropped on the front blocking layer in order to supply the dark current plus the background photocurrent as shown in Fig. 6.

\subsection{Experiment and Analysis for Photocurrent}

In order to understand the potential profile of the rear blocking layer observed by the photoelectrons, the spectral response and the voltage dependence of responsivity for a monochromatic radiation are both important experiments to proceed. The first one 


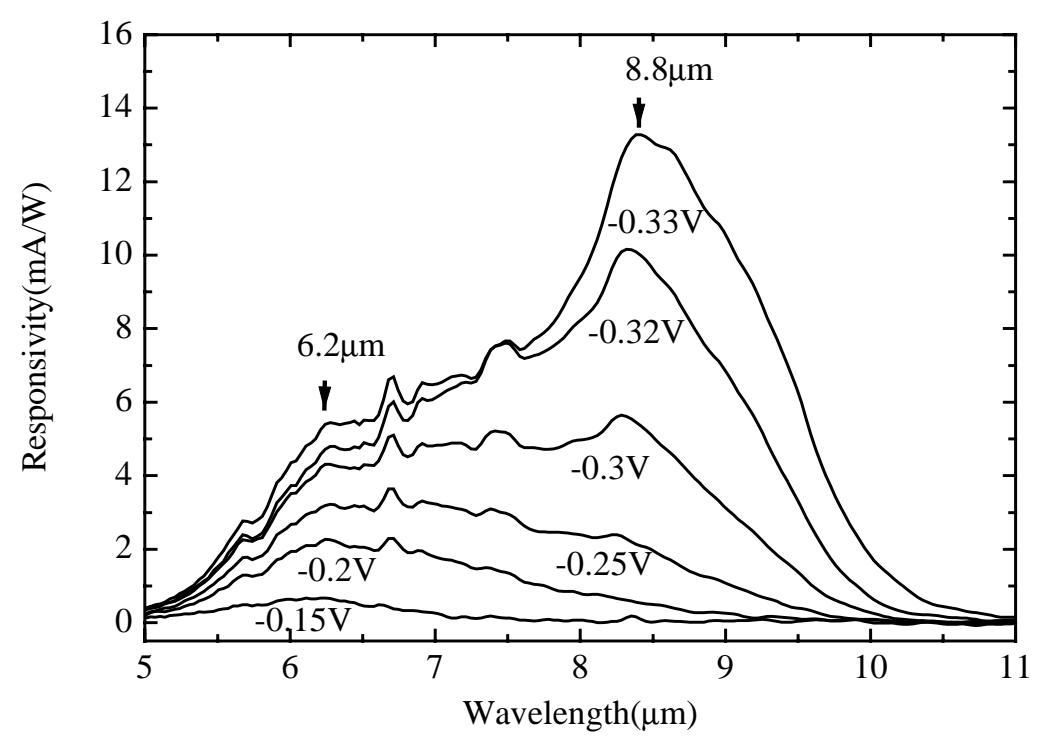

Fig. 7. Spectral response venues several bias at $30 \mathrm{~K}$.

is to identify the wavelength range in which the sample has a response. The latter is to observe the increment trend of photoelectrons versus bias due to a monochromatic radiation. With the experimental results and our formula, the potential profile of the rear blocking layer expects to be understood.

First, the spectral responsivity was measured at various bias and temperature by using Perkin Elmer 2000 Fourier transform infrared (FTIR) spectrometer. The absolute value of the responsivity was determined by measuring the blackbody responsivity $R$ with a blackbody source. In the experiment, the temperature of the blackbody source was set at $800 \mathrm{~K}$. The response

$R(800 \mathrm{~K})$ can be written as

$$
R(800 \mathrm{~K})=\frac{R_{P} \int_{0}^{\infty} R(\lambda) \cdot W(\lambda, 800 \mathrm{~K}) d \lambda}{\int_{0}^{\infty} W(\lambda, 800 \mathrm{~K}) d \lambda},
$$

where $W(\lambda, T)$ is the radiance of the blackbody source at $T, R(\lambda)$ is the relative responsivity normalized by the peak responsivity and measured by using FTIR and $R_{\mathrm{P}}$ is the peak responsivity of $R(\lambda)$, which is what we want to know.

Figure 7 shows spectral responsivity venues several biases at $30 \mathrm{~K}$. The primary peak position varies from the short wavelength to the large one with increasing the bias. Especially, the peak of the short wavelength $(6.2 \mu \mathrm{m})$ responsivity is going to appear at $-0.2 \mathrm{~V}$ and the peak of the long wavelength $(8.8 \mu \mathrm{m})$ responsivity is appearing at $-0.29 \mathrm{~V}$ in Fig. 7 . It indicates that the photoelectrons from the bottom edge of the second miniband can just pass the blocking layer at $-0.29 \mathrm{~V}$, i.e., the effective barrier height is about $207 \mathrm{meV}$. The short wavelength peak $(6.2 \mu \mathrm{m})$ and long wavelength peak $(8.8 \mu \mathrm{m})$ are close to our designed values $(6.0 \mu \mathrm{m}$ and $8.7 \mu \mathrm{m})$.

The voltage dependence of responsivity at various wavelengths is measured with a chopper and lock-in amplifier. The monochromatic infrared radiation is generated by passing the thermal radiation from a glow bar through a monochromator and a long-pass filter with cut-off wavelength $5 \mu \mathrm{m}$, and the photocurrent is transformed into voltage signal by a transconductance amplifier SR570. The measured voltage dependence of responsivity at wavelengths $6.6 \mu \mathrm{m}$ and $8.8 \mu \mathrm{m}$ is shown as open circle in Fig. 8. It is observed that the long wavelength responsivity is negligible at bias smaller than $-0.2 \mathrm{~V}$ in magnitude, however it increases more rapidly than the short wavelength one at high bias region. 


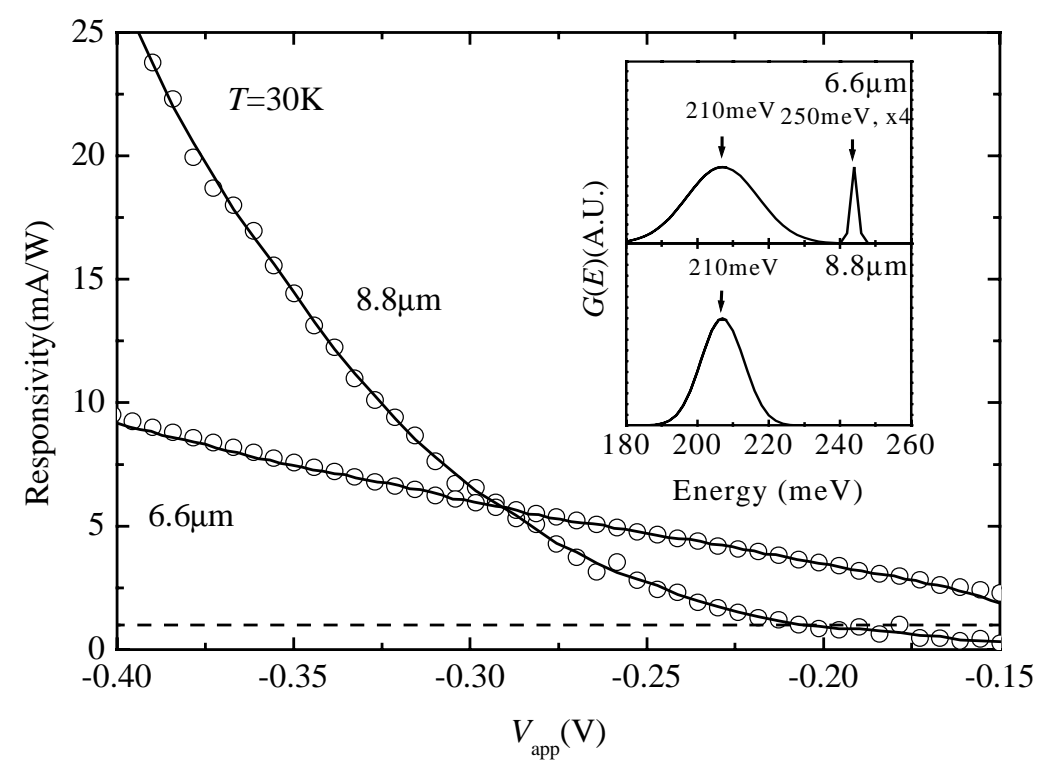

Fig. 8 Voltage dependence of responsivity at two peak wavelength $6.6 \mu \mathrm{m}$ and $8.8 \mu \mathrm{m}$ for experiment (scatter curve) and theory (solid curve).

In order to match the experimental data with Eq. (7), we have to figure out the voltage drop on the rear blocking layer. With background radiation incident upon the sample and at thermal equilibrium, no current should be detected in our sample. The barriers of the two blocking layers are expected to tilt up to block the photocurrent. Under this situation, the voltage drop on the rear blocking layer can be considered as negative while that on the front one as positive. The total amount of the voltage drop is zero. As the external bias increases the voltage drop on the rear blocking layer increases and is becoming positive. As a first-order approximation, the voltage drop on the rear blocking layer can be written as

$$
V_{\mathrm{r}}=V_{\text {off }}+b \times V_{\text {app }} \text {, }
$$

where $V_{\text {off }}$ is the negative voltage drop on the rear blocking layer at zero bias, and $b$ represents the percentage of external applied bias to drop on the rear blocking layer.

Another important factor in Eq. (7) is $G(E)$, which involves with the energy distribution of the photoelectrons. As expected, the photoelectrons may relax their energy and enter into the bottom state of the second miniband. Therefore, $G(E)$ can be approximated as a two-peak function for a monochromatic radiation except the transition between the top of the first miniband into the bottom of the second miniband.

Taken the $6.6-\mu \mathrm{m}$ radiation into consideration. The photoelectrons transit from the $62-\mathrm{meV}$ state in the first miniband to the $250-\mathrm{meV}$ state in the second miniband. In addition, relaxation may cause the photoelectrons to be accumulated in the bottom $(210-\mathrm{meV})$ state in the second miniband. In this case, $G(E)$ may be a two-peak function as that shown in the insert of Fig. 8. For the 8.8- $\mu \mathrm{m}$ radiation, $G(E)$ become a one-peak function since the final state is the bottom state of the second miniband. In the fitting, we use Gaussian function with the peak value and the associated variance as parameters to simulate a distribution in $G(E)$. Equation (9) is used to calculate the electric field $\mathcal{E}$ in $T(E, \varepsilon)$. The final results of the fitting are shown as the solid curve in Fig. 8. The insert shows $G(E)$ for $6.6-\mu \mathrm{m}$ and $8.8-\mu \mathrm{m}$ radiation.

For $6.6-\mu \mathrm{m}$ radiation, the contribution from the $250-\mathrm{meV}$ state is represented as the dash line. For these photoelectrons in $250-\mathrm{meV}$ state, they do not observe any barrier even at $-0.15 \mathrm{~V}$ and produce a saturated photocurrent at all bias range shown in Fig. 8. In fact, almost $90 \%$ of the photoelectrons are relaxed into the bottom state of the second miniband and cause the responsivity to increase with bias since they have to tunnel through the barrier just like the photoelectrons produced by $8.8 \mu \mathrm{m}$-radiation. 


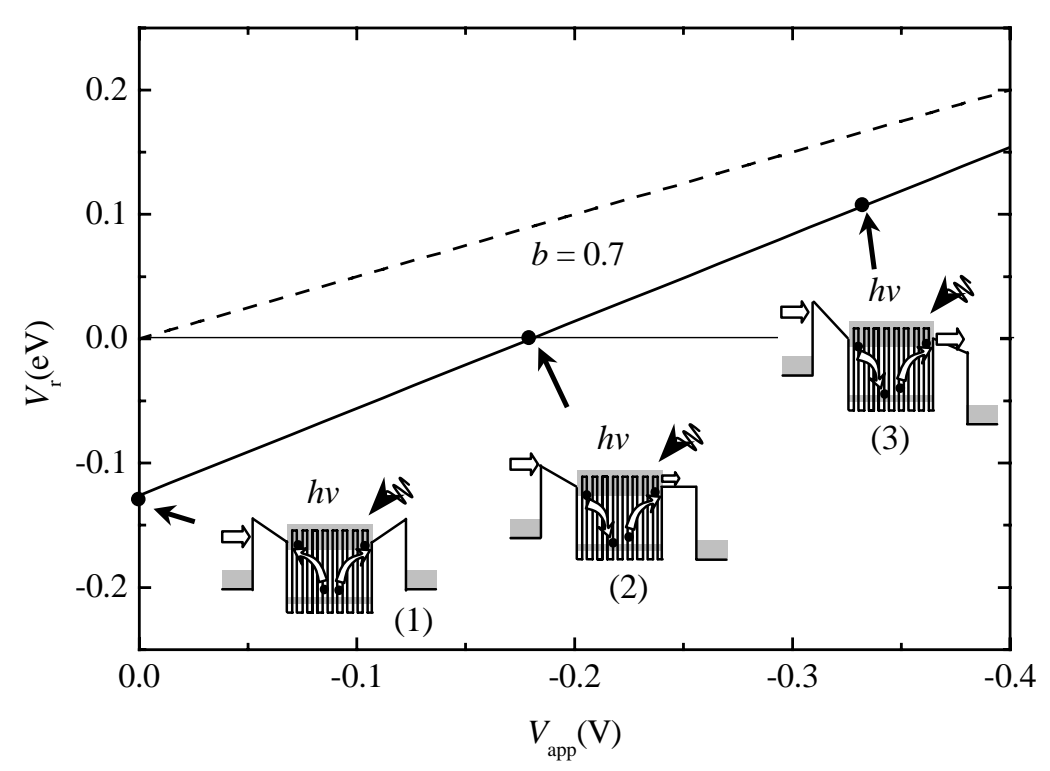

Fig. 9 Bias on the rear blocking layer with only dark current (dash line) and photocurrent (solid line) at 30K.

The voltage drop on the rear blocking layer versus the external bias with background radiation incident upon the sample is shown as the solid line in Fig. 9. The three inserts show the potential profile at three different biases. The dash line in the same figure stands for the same voltage drop with only dark current existing.

As shown in the first insert of Fig. 9, the blocking layers are tilted up for $126 \mathrm{meV}$ at zero bias due to the background radiation incident upon the detector. The second miniband is confined by the tilt-up blocking layer and no electron can tunnel through it. On the contrary, there is no voltage drop on the rear blocking layer with only the dark current existing.

As the external bias increases, $70 \%$ of the voltage is dropped on the rear blocking layer and attempts to decrease its barrier height. As shown in the second insert of Fig. 9, the rear blocking layer has a flat barrier at $-0.18 \mathrm{~V}$. It is noted that the barrier height of the blocking layer is $16.5 \mathrm{meV}$ higher than the bottom of the second miniband. In this case, only those photoelectrons with high enough energy can pass the blocking layer. In particular, those photoelectrons in the bottom state of the second miniband cannot tunnel through the blocking layer. As shown in the insert of Fig. 8, this corresponds to those photoelectrons without relaxation. The photocurrent is expected to be small since the number of the photoelectrons without relaxation is limited. As shown in Fig. 7 , at $-0.2 \mathrm{~V}$, the responsivity is small and only the short wavelength radiation can cause response. This is consistent with the prediction from the potential profile of the rear blocking layer.

As the external bias increases up to $-0.33 \mathrm{~V}$, the rear blocking layer is tilted down for $105 \mathrm{meV}$. The electric field on the barrier is $2.1 \times 10^{6} \mathrm{~V} / \mathrm{cm}$. The transmission probability for the photoelectrons at the bottom state of the second miniband through the blocking layer is about 0.13 . It increases dramatically for the photoelectrons with higher energy. Therefore, the blocking layer seems to be transparent to all photoelectrons, and the responsivity in Fig. 7 shows high values and wide range of responded wavelength.

\section{CONCLUSION}

We have investigated the characteristics of the background photocurrent and dark current in a superlattice infrared photodetector, which has a 20-period $\mathrm{GaAs}(6 \mathrm{~nm}) / \mathrm{Al}_{0.28} \mathrm{Ga}_{0.72} \mathrm{As}(4 \mathrm{~nm})$ superlattice embedded between two $50 \mathrm{~nm} \mathrm{Al}_{0.28} \mathrm{Ga}_{0.72} \mathrm{As}$ block layers. It is concluded from the dark-current $I-V$ characteristics and photo responsivity versus external bias that the external bias is indeed redistributed due to the background photocurrent. We successfully use a simple model to describe the physical mechanisms of the dark current and photo responsivity. With infrared radiation incident upon the sample, there are almost $70 \%$ of $V_{\text {app }}$ is dropped on the rear blocking layer to decrease the barrier height of the blocking layer. Especially at zero bias, the back blocking layer is tilted up for $126 \mathrm{meV}$ to prevent from the background photocurrent and dark current. In 
addition, the photo responsivity due to the short wavelength excitation indicates that the photoelectrons do have the inelastic relaxation of the energy along the growth direction.

\section{ACKNOWLEDGE}

This project is sponsored partially by National Science Council under grant number NSC-89-2115-E-002-048.

\section{REFERENCE}

1. L. C. West and S. J. Eglash, "First observation of an extremely large dipole infrared transition within the conduction band of a GaAs quantum well," Appl. Phvs. Lett., vol. 46, pp. 1156-1159, 1983.

2. S. R. Andrews and B. A. Miller, "Experimental and theoretical studies of the performance of quantum-well infrared photodetectors," J. Appl. Phys., vol. 70, pp. 993-1003, 1991.

3. B. F. Levine, "Quantum-well infrared photodetectors," J. Appl. Phys., vol. 74, pp. R1-R81, 1993.

4. G. Sarusi, S. D. Gunapala, J. S. Park, and B. F. Levine, "Design and per-formance of very long-wavelength $\mathrm{GaAs}_{\mathrm{A}} / \mathrm{Al}_{\mathrm{x}} \mathrm{Ga}_{1-\mathrm{x}}$ As quantum-well infrared photodetectos," J. Appl. Phys., vol. 76, pp. 6001-6008, 1994.

5. K.K. Choi, "The physics of quantum well infrared photodetector", World Scientific, chap. 2, 1997.

6. G.Hasnain, B.F.Levine, S.Gunapala, and N.Chand, "Large photoconductive gain in quantum well infrared photodetectors", Appl. Phys. Lett., vol. 57, pp. 608-610, 1990.

7. E. Pelve et al., "Analysis of the dark current in doped-well multiple quantum well AlGaAs infrared photodetectors", J. Appl. Phys., vol. 66, pp. 5656-5659, 1989.

8. C. H. Kuan et al., "Hot-electron distribution in multiple quantum well infrared photodetectors", Appl. Phys. Lett., vol. 63, pp.2091-2093, 1993.

9. P. Man and D. S. Pan, "Hot-carrier-temperature model for the dark current of quantum-well infrared photodetectors", Appl. Phys. Lett., vol. 66, pp. 192-194, 1995.

10. D. F. Nelson, R. C. Milier, and D. A. Kleinman, "Band-nonparabolicity effects in semiconductor quantum wells", Phys. Rev. B, vol. 35, pp.7770-7773, 1987.

11. M. Helm, "Infrared spectroscopy and transport of electrons in semiconductor superlattices", Semicond. Sci. Technol., vol.10, pp.557-575, 1995.

12. B. F. Levine, C. G. Bethea, G. Hasnain, V. O. Shen, E. Pelve, R. R. Abbott, and S. J. Hsieh, "High sensitivity low dark current $10 \mu \mathrm{m}$ GaAs quantum well infrared photodetectors", Appl. Phys. Lett., vol. 56, pp. 851-853, 1990.

13. H. C. Liu, Li Jianmeng, M. Buchanan, and Wasilewski, Z.R., "High-frequency quantum-well infrared photodetectors measured by microwave-rectification technique", IEEE J. Quantum Electronics, vol. 32, pp.1024 -1028, 1996.

14. H.C.Liu et al., "Quantum well intersubband transition physics and devices", A Harcourt Science \& Technology Co., pp. 137-146, 2000. 